\title{
Clinical Application of Fetal Umbilical Vein Doppler Parameters in Maternal Preeclampsia
}

\author{
Li Sheng*, Wei Xu, Jiehao Huang \\ The First Hospital Affiliated to Yangtze University, Jingzhou First People’s Hospital, Jingzhou, China \\ Email: *ku80@sina.com
}

How to cite this paper: Sheng, L., Xu, W. and Huang, J.H. (2021) Clinical Application of Fetal Umbilical Vein Doppler Parameters in Maternal Preeclampsia. Yangtze Medicine, 5, 295-302.

https://doi.org/10.4236/ym.2021.54027

Received: October 11, 2021

Accepted: December 25, 2021

Published: December 28, 2021

Copyright ( 2021 by author(s) and Scientific Research Publishing Inc. This work is licensed under the Creative Commons Attribution International License (CC BY 4.0).

http://creativecommons.org/licenses/by/4.0/

(c) (i) Open Access

\begin{abstract}
Objective: To observe the changes of fetal umbilical vein (UV) Doppler parameters in pregnant women with preeclampsia (PE) and analyze their predictive values for maternal PE. Methods: Forty-six patients with PE who underwent systematic ultrasound examination in our hospital from December 2017 to May 2021 were included as the subjects, which were divided into two groups according to the severity of the disease ( 23 cases in each group). And 120 normal pregnant women who underwent health examination in our hospital during the same period were enrolled as the control group. Color Doppler ultrasonography was used to monitor the umbilical vein flow (QUV), left portal vein flow (QLPV), venous catheter flow (QDV), left portal vein (LPV) shunt rate and venous catheter (DV) shunt rate. And the sensitivity and specificity of the related indexes were calculated and analyzed according to the gold standard for clinical diagnosis of PE. Results: As the severity of PE increased, the fetal QUV, QLPV and LPV shunt rates decreased, and the QDV and DV shunt rates increased, with statistically significant differences compared with the control group $(\mathrm{P}<0.05)$. The specificity and sensitivity of the combination of fetal QUV, QLPV, QDV, LPV shunt rate and DV shunt rate in predicting $\mathrm{PE}$ were higher than those of the indexes used alone $(\mathrm{P}<0.05)$. Conclusion: The fetal umbilical vein Doppler parameters QUV, QLPV, QDV, LPV shunt rate, and DV shunt rate have some value in predicting PE, but their combination showed greater value, as well as higher diagnostic and clinical significance.
\end{abstract}

\section{Keywords}

Fetal Umbilical Vein, Doppler Parameters, Preeclampsia

\section{Introduction}

Preeclampsia (PE) is a pregnancy-related condition characterized by changes in 
clinical signs and symptoms such as increased blood pressure and proteinuria that appear after 20 weeks of gestation in women [1]. The disease causes different degrees of alterations in and damage to the cardiac, renal, hepatic, neurological and other organ systems of pregnant women. And it can damage the placenta-fetus, and seriously affect maternal and fetal health, and therefore it is a major cause of clinical maternal and fetal death [2]. The pathogenesis of PE is complex, and there is no effective and safe treatment method. Therefore, timely, accurate and effective monitoring and prevention are of great clinical value. As the umbilical vein is the only oxygen supply pathway to the fetus, along with the increase of gestational weeks, the fetal oxygen demand will increase due to the needs of fetal growth and development, and its blood flow will increase accordingly. In contrast, patients with PE have abnormal blood perfusion to the placenta due to suppressed trophoblast infiltration, impaired spiral artery remodeling, vasospasm and placental vascular endothelial cell dysfunction [3]. When the fetus is exposed to inadequate blood and oxygen supply to the uteroplacenta, some hemodynamic changes occur in the umbilical venous circulation to counteract the inadequate oxygen supply [4]. In this study, we analyzed the efficacy of fetal umbilical vein Doppler parameters in the prediction of PE through a controlled clinical study, which is reported below.

\section{Data and Methods}

\subsection{General Data}

Forty-six patients with PE who were treated in the obstetrics department of our hospital from December 2017 to May 2021 were selected for the study, and were divided into mild and severe groups based on the degree of PE, with 23 cases in each group. The gestational weeks at the time of the proposed initial diagnosis were between 16 - 20 weeks. Inclusion criteria: 1) all had regular antenatal checkups at our hospital; 2) 18 - 35 years old with $\geq 20$ weeks of gestational cycle; 3) intrauterine singleton pregnancy; 4) meeting the diagnostic criteria and severity classification criteria of PE [5]. Mild: blood pressure $\geq 140 / 90 \mathrm{mmHg}$ combined with proteinuria $>300 \mathrm{mg} / 24 \mathrm{~h}$, may be accompanied by headache and abdominal discomfort; severe: blood pressure $\geq 160 / 110 \mathrm{mmHg}$, proteinuria $>2$ $\mathrm{g} / 24 \mathrm{~h}$, blood creatinine $>106 \mu \mathrm{mol}$, oliguria. 5) All were delivered of a baby in our hospital. Exclusion criteria: a) combined with other high-risk pregnancy factors, such as age, diabetes mellitus, birth canal abnormalities, previous history of adverse pregnancy and delivery; b) fetal malformations; c) incomplete clinical data. One hundred and twenty healthy pregnant women who were registered at the obstetrics department of our hospital and received regular prenatal checkups during the same period were used as the control group, all of whom were free of chronic hypertension, anemia and other cardiovascular diseases.

\subsection{Methods}

A GE Voluson E8 color Doppler ultrasound diagnostic instrument with a two- 
dimensional 4C-D convex array probe (frequency 1.5 - 4.6 MHz) was applied. Color Doppler ultrasonography was performed on the fetuses in both groups, and the pregnant women were instructed to take the supine position, select the intra-abdominal segment UV, keep the angle between the ultrasound beam and the long axis of blood vessels $0^{\circ}-30^{\circ}$, Doppler sampling volume $2 \mathrm{~mm}$, and show the standard plane of abdominal circumference on the transverse section of fetal abdomen. If the mother felt uncomfortable during the examination, stop the examination immediately and ask her to lie on her side or rest for a while before performing the examination. The fetal images were acquired at rest, frozen after satisfactory images were obtained, and the parameters of the umbilical venous circulation, such as QUV, QLPV, DV, QDV, QLPV/QUV (LPV shunt rate) and QDV/QUV (DV shunt rate), were analyzed by the same senior ultrasonographer.

Specific operations: 1) UV: the standard plane of abdominal circumference was shown on the transverse section of the fetal abdomen, and the UV was seen entering the liver along the anterior abdominal wall of the fetus, and the UV between the UV into the abdominal cavity more than $2 \mathrm{~mm}$ and the venous catheter was selected; 2) LPV: after the UV was found in the standard section of the abdomen, the first branch toward the left lobe of the liver was found along its course, and the position of the root about $2 \mathrm{~mm}$ was selected, and the internal diameters of the UV and LPV were measured respectively, and then according to the formula. $\mathrm{Q}=\pi \times(\mathrm{D} / 2)^{2} \times 0.5 \times \mathrm{Vmax}[6]$ to calculate the QUV, QLPV, and LPV shunt rates, respectively. 3) The end branches of the umbilical vein were traced along the umbilical vein alignment in selected oblique transverse sections until the umbilical vein catheter could be observed, its internal diameter was measured, and the sampling volume was set at the umbilical vein catheter to obtain the maximum fetal pulse Doppler spectrum in the quiet state, and the maximum fetal cardiac systolic blood flow velocity was measured, and then the blood flow rate of the umbilical vein catheter QDV was calculated according to the formula: 0.7 Vmax- $\mathrm{dv} \times(\mathrm{DDV} / 2)^{2} \times \pi$ [7], where Vmax- $\mathrm{dv}$ is the maximum flow velocity of the venous catheter and DDV is the internal diameter of the umbilical venous catheter. Color Doppler flow imaging was obtained, and the pulse Doppler sampling volume was placed at the same site with a sampling volume of $2 \mathrm{~mm}$, and the angle between the sampling line and the direction of blood flow was controlled at $0^{\circ}-30^{\circ}$, and the maximum flow velocity (Vmax) was measured, and then the LPV shunt rate and DV shunt rate were calculated.

\subsection{Statistical Analysis}

This study was analyzed and processed by SPSS22.0 software. Count data were expressed as number of cases (\%), with 2-test used for comparison between groups; Measurement data were described as $(\bar{x} \pm s)$, with LSD-t test used for comparison between groups. Clinical diagnosis was used as the gold standard to analyze the sensitivity and specificity of relevant indexes for predicting PE, with $\mathrm{P}<0.05$ indicating significantly statistical differences. 


\section{Results}

\subsection{Comparison of Basic Clinical Conditions of Pregnant Women between Groups}

The analysis of clinical data showed that there was no significant difference in age, pre-pregnancy body mass index (BMI), gestational week of examination, pregnancy and delivery between PE mild group, PE severe group, and the control group, with no statistical significance $(P>0.05)$. The detailed results are shown in Table 1.

\subsection{Comparison of Fetal Umbilical Vein Circulation Doppler Parameters between Groups}

The results of clinical Doppler examination showed that in the PE group, the fetal QUV, QLPV and LPV shunt rates were lower than those in the control group, while the QDV and DV shunt rates were higher than those in the control group. Both of the mild and severe PE groups showed statistically significant differences, compared with the normal control group $(\mathrm{P}<0.05)$. As the severity of PE increased, the fetal QUV, QLPV and LPV shunt rates were lower, while the shunt rates of QDV and DV were higher in the severe group, compared with the mild group, all with statistical significance $(\mathrm{P}<0.05)$. The results are shown in Table 2.

Table 1. Comparison of the basic conditions of pregnant women in the PE and control groups $(\bar{x} \pm s)$.

\begin{tabular}{ccccc}
\hline Item & $\begin{array}{c}\text { Control group } \\
(\mathbf{n}=120)\end{array}$ & $\begin{array}{c}\text { PE mild group } \\
(\mathbf{n}=\mathbf{2 3})\end{array}$ & $\begin{array}{c}\text { PE severe group } \\
(\mathbf{n}=\mathbf{2 3})\end{array}$ & P-value \\
\hline Age (years) & $25.67 \pm 3.29$ & $26.61 \pm 4.08$ & $26.92 \pm 4.11$ & 0.175 \\
$\begin{array}{c}\text { Pre-pregnancy BMI } \\
\text { Week of pregnancy }\end{array}$ & $21.64 \pm 2.80$ & $22.08 \pm 2.93$ & $22.10 \pm 2.84$ & 0.083 \\
$\begin{array}{c}\text { examined (weeks) } \\
\text { Pregnancy times (times) }\end{array}$ & $1.96 \pm 0.22$ & $2.03 \pm 0.28$ & $1.98 \pm 0.27$ & 0.273 \\
$\begin{array}{c}\text { Number of births (times) } \\
\text { Number }\end{array}$ & $0.51 \pm 0.11$ & $0.49 \pm 0.23$ & $0.49 \pm 0.17$ & 0.109 \\
\hline
\end{tabular}

Note: $\mathrm{P}>0.05$ compared with normal control group.

Table 2. Comparison of fetal umbilical vein blood circulation Doppler ultrasound parameters indexes in each group $(\bar{x} \pm s)$.

\begin{tabular}{ccccccc}
\hline Group & $\begin{array}{c}\text { Number } \\
\text { of cases }\end{array}$ & $\begin{array}{c}\text { QLPV } \\
(\mathrm{ml} / \mathrm{min})\end{array}$ & $\begin{array}{c}\text { QDV } \\
(\mathrm{ml} / \mathrm{min})\end{array}$ & $\begin{array}{c}\text { QUV } \\
(\mathrm{ml} / \mathrm{min})\end{array}$ & $\begin{array}{c}\text { LPV shunt } \\
\text { rate }\end{array}$ & $\begin{array}{c}\text { DV shunt } \\
\text { rate }\end{array}$ \\
\hline Control group & 120 & $46.21 \pm 12.17$ & $39.05 \pm 12.18$ & $141.83 \pm 30.51$ & $0.33 \pm 0.04$ & $0.22 \pm 0.04$ \\
PE mild group & 23 & $39.17 \pm 8.26$ & $55.74 \pm 10.62$ & $112.24 \pm 40.22$ & $0.24 \pm 0.05$ & $0.34 \pm 0.11$ \\
PE severe group & 23 & $28.37 \pm 6.30$ & $60.82 \pm 10.53$ & $108.36 \pm 32.22$ & $0.17 \pm 0.10$ & $0.42 \pm 0.07$ \\
$\begin{array}{c}t \text {-value } \\
P \text {-value }\end{array}$ & & 9.065 & 6.125 & 10.811 & 3.251 & 4.236 \\
& & 0.000 & 0.000 & 0.001 & 0.000 & 0.000 \\
\hline
\end{tabular}




\subsection{Fetal Umbilical Vein Blood Circulation Doppler Parameters to Predict PE Efficacy}

Clinical Doppler findings showed that using fetal QUV, QLPV, QDV, LPV shunt rate, and DV shunt rate alone had higher specificity and sensitivity than the combination of them in pregnant women with $\mathrm{PE}$, with statistical significance $(\mathrm{P}<0.05)$. The results are shown in Table 3.

\section{Discussion}

$\mathrm{PE}$ is a disease specific to pregnancy with a prevalence of about $5 \%-10 \%$ [8]. Clinical manifestations are hypertension, proteinuria or other multisystem abnormalities after 20 weeks of gestation. The condition is an important contributor to maternal and fetal morbidity and mortality [9]. Therefore, early prediction, early intervention and timely monitoring of PE are particularly important. With the continuous innovation and development of color Doppler ultrasound diagnostic technology, its role in prenatal diagnosis is increasing. Due to its many advantages over other examinations [10], such that it is safe, noninvasive, accurate, reproducible, and can reflect the blood supply of tissues and organs in real time, it has been used in the evaluation of PE [11].

At present, the clinical pathogenesis of $\mathrm{PE}$ is not fully clarified, but it has been widely recognized clinically that the placenta is a key factor in the pathogenesis of PE [12]. Decreased trophoblast infiltration capacity in the placenta of PE, shallow placental implantation, and impaired uterine spiral artery remodeling lead to decreased vascular production in the placenta and ultimately to inadequate placental perfusion. When the placenta is undersupplied with blood and oxygen, changes in hormone levels in the body contribute to an increase in the internal diameter of the DV, which leads to an increase in DV shunting, while extensive constriction of the intrahepatic portal system occurs, and UV blood is redistributed, preferentially flowing through the venous catheter away from the liver and towards the heart, reaching the coronary arteries and cerebral circulation, etc., which facilitates the nutrition and oxygen requirements of vital organs [13].

Table 3. Comparison of the efficacy of single and combined prediction of fetal umbilical vein circulation Doppler parameters for PE.

\begin{tabular}{ccc}
\hline Indicator & Sensitivity (\%) & Specificity (\%) \\
\hline QUV & $75.43^{\mathrm{a}}$ & $74.50^{\mathrm{a}}$ \\
QLPV & $80.40^{\mathrm{a}}$ & $73.45^{\mathrm{a}}$ \\
QDV & $85.90^{\mathrm{a}}$ & $85.50^{\mathrm{a}}$ \\
LPV Shunt Rate & $75.8^{\mathrm{a}}$ & $73.20^{\mathrm{a}}$ \\
DV Shunt Rate & $84.90^{\mathrm{a}}$ & $86.45^{\mathrm{a}}$ \\
Joint prediction & 95.40 & 98.15 \\
\hline
\end{tabular}

Note: Comparison with joint predicted $\mathrm{PE}$, ${ }^{\mathrm{a}} \mathrm{P}<0.05$. 
The umbilical vein is an important channel of fetal blood circulation, supplying highly oxygenated blood flow from the placenta to the fetus, and is responsible for obtaining the nutrients required for fetal growth and development. During normal pregnancy, along with the maturation of the placenta, the placental vessels gradually thicken, the vascular resistance decreases, and the ventricular compliance also increases with the decrease of cardiac after load, which results in the decrease of end-diastolic pressure and the increase of systolic return flow rate; meanwhile, the internal diameter of the DV increases gradually with the prolongation of the gestational week, and the UV blood flow increases accordingly. Thus, normal fetal UV blood flow parameters indicators increase with increasing gestational weeks. Based on this, the present study attempted to predict the occurrence of PE by detecting fetal UV blood circulation parameters, and then provide guidance and basis for early clinical intervention.

Clinical studies have found that umbilical vein blood flow predicts fetal growth restriction [14] [15] as well as infants younger than gestational age [16] and can be used as one of the indicators for fetal birth risk classification [17]. Because PE patients are less frequent clinically, they are often overlooked in clinical diagnosis, but they are significantly correlated with fetal prognosis, and analyzing the correlation between changes in UV circulatory Doppler parameters and different degrees of preeclampsia is a simple, noninvasive, reproducible and feasible method for predicting intrauterine distress and hypoxia, which is more sensitive and accurate than the currently used fetal monitoring, and it can quantify the amount of UV circulatory perfusion, promptly It is important to reduce the rate of perinatal disability and improve the quality of perinatal medicine and eugenics.

The results of this study showed that compared with the control group, fetuses in PE groups had significantly higher QDV and DV shunt rate, and significantly lower QUV, QLPV and LPV shunt rate. In addition, the severity of PE showed positive associations with QDV and DV shunt rate, and negative associations with QUV, QLPV and LPV shunt rate. In normal pregnancies, the effect of QUV on DV shunts is the greatest, because the placental hormonal effect thickens the umbilical vein, causing an increase in blood flow velocity and flow rate, which in turn increases the DV shunt rate. In addition, the specificity and sensitivity of the combined parameters of fetal QUV, QLPV, QDV, LPV shunt rate, and DV shunt rate in predicting $\mathrm{PE}$ were $95.40 \%$ and $98.15 \%$, respectively, which were higher than those of single indicators $(\mathrm{P}<0.05)$.

\section{Conclusion}

In conclusion, the fetal umbilical vein Doppler parameters QUV, QLPV, QDV, LPV shunt rate, and DV shunt rate change significantly in pregnant women with PE, which have some clinical significance in PE prediction. We found that the combination has higher clinical value, which can be used in clinical practice. 


\section{Conflicts of Interest}

The authors declare no conflicts of interest regarding the publication of this paper.

\section{References}

[1] Chen, H.F. (2020) Correlation Analysis of Fetal Heart Monitoring, Fetal Umbilical Artery and Middle Cerebral Artery Flow Doppler Ultrasound Parameters in Late Pregnancy and Perinatal Prognosis in Preeclampsia. China Maternal and Child Health Care, 10, 1852-1855.

[2] Li, J.H., Liu, Z., Wu, S.Y., et al. (2016) Prognostic Analysis of Venous Catheter, Umbilical Vein and Umbilical Artery and Middle Cerebral Artery Blood Flow Detection in Preeclamptic Fetuses. Modern Advances in Obstetrics and Gynecology, 1, 29-33.

[3] Boeldt, D.S. and Bird, I.M. (2017) Vascular Adaptation in Pregnancy and Endothelial Dysfunction in Preeclampsia. Journal of Endocrinology, 232, R27-R44. https://doi.org/10.1530/JOE-16-0340

[4] Goldman-Wohl, D. and Yagel, S. (2002) Regulation of Trophoblast Invasion: From Normal Implantation to Pre-Eclampsia. Molecular and Cellular Endocrinology, 187, 233-238. https://doi.org/10.1016/S0303-7207(01)00687-6

[5] Yang, Z. and Zhang, W.Y. (2020) Interpretation of the Guidelines for the Diagnosis and Treatment of Hypertensive Disorders in Pregnancy. Chinese Journal of Obstetrics and Gynecology, 6, 425-432.

[6] Kiserud, T., Rasmussen, S. and Skulstad, S. (2000) Blood Flow and the Degree of Shunting Through the Ductus Venosus in the Human Fetus. American Journal of Obstetrics \& Gynecology, 182, 147-153. https://doi.org/10.1016/S0002-9378(00)70504-7

[7] Barbera, A., Galan, H.L., Ferrazzi, E., Rigano, S., Józwik, M., Battaglia, F.C. and Pardi, G. (1999) Relationship of Umbilical Vein Blood Flow to Growth Parameters in the Human Fetus. American Journal of Obstetrics \& Gynecology, 181, 174-179. https://doi.org/10.1016/S0002-9378(99)70456-4

[8] Freeman, R.K. (2008) Antepartum Testing in Patients with Hypertensive Disorders in Pregnancy. Seminars in Perinatology, 32, 271-273. https://doi.org/10.1053/j.semperi.2008.04.009

[9] Ieyabalan, A. and Conrad, K.P. (2007) Renal Function during Normal Pregnancy and Preeelampsia. Frontiers in Bioscience, 12, 2425-2437.

https://doi.org/10.2741/2244

[10] Picklesimer, A.H., Oepkes, D., Moise, K.J., et al. (2007) Determinants of the Middle Cerebral Artery Peak Systolic Velocity in the Human Fetus. American Journal of Obstetrics \& Gynecology, 197, 526.E1-526.E4.

https://doi.org/10.1016/j.ajog.2007.04.002

[11] Armant, D.R., Fritz, R., Kilburn, B.A., Kim, Y.M., Nien, J.K., Maihle, N.J., Romero, R. and Leach, R.E. (2015) Reduced Expression of the Epidermal Growth Factor Signaling System in Preeclampsia. Placenta, 36, 270-278. https://doi.org/10.1016/j.placenta.2014.12.006

[12] Black, K.D. and Horowitz, J.A. (2018) Inflammatory Markers and Preeclampsia: A Systematic Review. Nursing Research, 67, 242-251. https://doi.org/10.1097/NNR.0000000000000285

[13] Dikshit, S. (2011) Fresh Look at the Doppler Changes in Pregnancies with Placen- 
tal-Based Complications. Journal of Postgraduate Medicine, 57, 138-140. https://doi.org/10.4103/0022-3859.81880

[14] Liao, T.B., Nomura, R.M., Liao, A.W., et al. (2014) Fetal Venous Circulation in Monochorionic Twin Pregnancies with Placental Insufficiency: Prediction of Acidemia at Birth or Intrauterine Fetal Death. Ultrasound in Obstetrics \& Gynecology, 43, 426-431. https://doi.org/10.1002/uog.12549

[15] To, W.W.K. and Mok, C.K.M. (2009) Fetal Umbilical Arterial and Venous Doppler Measurements in Gestational Diabetic and Nondiabetic Pregnancies near Term. The Journal of Maternal-Fetal \& Neonatal Medicine, 22, 1176-1182. https://doi.org/10.3109/14767050903042546

[16] Parra-Saavedra, M., Crovetto, F., Triunfo, S., Savchev, S., Parra, G., Sanz, M., Gratacos, E. and Figueras, F. (2013) Added Value of Umbilical Vein Flow as a Predictor of Perinatal Outcome in Term Small-for-Gestational-Age Fetuses. Ultrasound in Obstetrics \& Gynecology, 42, 189-195. https://doi.org/10.1002/uog.12380

[17] Prior, T., Mullins, E., Bennett, P., et al. (2014) Umbilical Venous Flow Rate in Term Fetuses: Can Variations in Flow Predict Intrapartum Compromise? American Journal of Obstetrics \& Gynecology, 210, 61.El-61.E8.

https://doi.org/10.1016/j.ajog.2013.08.042 